Supplement of Hydrol. Earth Syst. Sci., 22, 4047-4060, 2018

https://doi.org/10.5194/hess-22-4047-2018-supplement

(c) Author(s) 2018. This work is distributed under

the Creative Commons Attribution 4.0 License.

(c) (1)

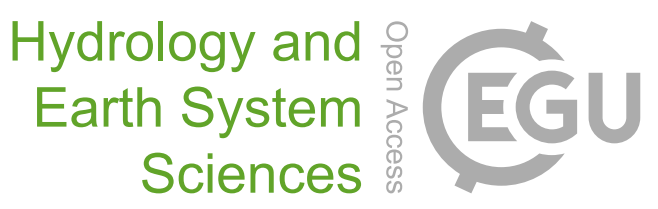

Supplement of

\title{
Hydrological effects of climate variability and vegetation dynamics on an- nual fluvial water balance in global large river basins
}

\section{Jianyu Liu et al.}

Correspondence to: Qiang Zhang (zhangq68@bnu.edu.cn)

The copyright of individual parts of the supplement might differ from the CC BY 4.0 License. 
Table S1. The validated parameter of eq. (14) and simulation accuracy of $R$ based on the estimated $n$ with the validated parameters for each basin

\begin{tabular}{|c|c|c|c|c|c|c|c|}
\hline \multirow[t]{2}{*}{ ID } & \multirow[t]{2}{*}{ Validated basin } & \multicolumn{3}{|c|}{ Model coefficients } & \multicolumn{3}{|c|}{$R$ simulation accuracy } \\
\hline & & $a$ & $b$ & $c$ & $R^{2}$ & RMSE & MAE \\
\hline 1 & Amur & 5.05 & -0.36 & -0.05 & 0.90 & 32.7 & 26.4 \\
\hline 2 & Aral & $0.39^{*}$ & $0.77^{*}$ & 0.04 & 0.80 & 7.9 & 6.1 \\
\hline 3 & Columbia & $0.58^{* * *}$ & 0.47 & -0.06 & 0.27 & 12.2 & 9.9 \\
\hline 4 & Congo & 0.92 & 0.10 & $-0.36^{* * *}$ & 0.94 & 12.8 & 10.7 \\
\hline 5 & Danube & 0.42 & 0.81 & -0.21 & 0.22 & 39.4 & 35.4 \\
\hline 6 & Indigirka & 0.02 & $2.37^{* * * *}$ & -0.02 & 0.85 & 16.1 & 13.1 \\
\hline 7 & Indus & $0.26^{*}$ & 0.57 & $0.59^{*}$ & 0.82 & 11.6 & 9.3 \\
\hline 8 & Kolyma & $0.34^{*}$ & $0.98^{* *}$ & -0.20 & 0.60 & 19.2 & 16.5 \\
\hline 9 & Lena & $0.39^{* * * *}$ & $0.71^{* *}$ & 0.19 & 0.84 & 9.0 & 7.1 \\
\hline 10 & Mackenzie & $0.28^{*}$ & $0.91^{* *}$ & -0.04 & 0.95 & 6.3 & 5.2 \\
\hline 11 & Mississippi & $1.06^{*}$ & -0.03 & 0.00 & 0.81 & 8.9 & 6.9 \\
\hline 12 & Niger & 0.03 & $2.22^{*}$ & 0.03 & 0.63 & 24.3 & 18.3 \\
\hline 13 & Nile & $0.99^{* * *}$ & -0.17 & 0.06 & 0.80 & 14.1 & 10.4 \\
\hline 14 & Northern Dvina & $1.53^{*}$ & -0.33 & -0.01 & 0.64 & 10.8 & 8.9 \\
\hline 15 & $\mathrm{Ob}$ & 0.34 & 0.61 & $0.30^{* *}$ & 0.85 & 14.3 & 11.5 \\
\hline 16 & Olenek & 0.33 & $0.76^{*}$ & 0.10 & 0.82 & 10.3 & 8.4 \\
\hline 17 & Parana & $0.35^{* * *}$ & $0.60^{*}$ & 0.39 & 0.76 & 11.4 & 8.3 \\
\hline 18 & Pearl & 2.90 & -0.10 & $-0.16^{* *}$ & 0.80 & 15.7 & 12.9 \\
\hline 19 & Pechora & 0.09 & $1.40^{*}$ & -0.01 & 0.97 & 21.8 & 17.2 \\
\hline 20 & Senegal & 0.44 & 0.44 & 0.06 & 0.87 & 16.5 & 13.1 \\
\hline 21 & Volga & $1.48^{* * *}$ & -0.04 & $-0.41^{*}$ & 0.82 & 4.0 & 3.3 \\
\hline 22 & Yangtze & 0.29 & 0.87 & -0.02 & 0.76 & 13.4 & 10.3 \\
\hline 23 & Yellow & 0.45 & 0.30 & -0.06 & 0.92 & 19.3 & 15.6 \\
\hline 24 & Yenisei & 0.86 & 0.28 & -0.01 & 0.58 & 11.0 & 9.1 \\
\hline 25 & Yukon & 0.32 & $0.79^{*}$ & 0.02 & 0.80 & 6.0 & 4.7 \\
\hline \multirow[t]{2}{*}{26} & Amur & 0.13 & 1.06 & 0.12 & 0.43 & 16.4 & 14.4 \\
\hline & All basins & $0.29^{* * *}$ & $0.86^{* * *}$ & $-3.3^{* * *}$ & 0.92 & 68.2 & 45.8 \\
\hline
\end{tabular}

'*', '**' and '***' represent the validated parameter are significant at the level of $p=0.1, p=0.05$ and $p=0.01$, respectively. 
Table S2. The validated parameter for the cross-variation of $n$.

\begin{tabular}{cccccccccc}
\hline ID & $\begin{array}{c}\text { Validated } \\
\text { basin }\end{array}$ & $a$ & $b$ & $c$ & ID & Validated basin & $a$ & $b$ & $c$ \\
\hline 1 & Amur & $0.28^{* * *}$ & $0.88^{* * *}$ & $-0.32^{* * *}$ & 14 & Northern Dvina & $0.29^{* * * *}$ & $0.86^{* * *}$ & $-0.33^{* * *}$ \\
2 & Aral & $0.28^{* * *}$ & $0.87^{* * *}$ & $-0.33^{* * *}$ & 15 & Ob & $0.28^{* * * *}$ & $0.90^{* * * *}$ & $-0.29^{* * * *}$ \\
3 & Columbia & $0.27^{* * * *}$ & $0.90^{* * * *}$ & $-0.31^{* * *}$ & 16 & Olenek & $0.29^{* * * *}$ & $0.86^{* * *}$ & $-0.33^{* * *}$ \\
4 & Congo & $0.29^{* * * *}$ & $0.86^{* * *}$ & $-0.32^{* * *}$ & 17 & Parana & $0.28^{* * * *}$ & $0.87^{* * *}$ & $-0.33^{* * *}$ \\
5 & Danube & $0.29^{* * *}$ & $0.88^{* * *}$ & $-0.13^{* * *}$ & 18 & Pearl & $0.32^{* * *}$ & $0.77^{* * *}$ & $-0.36^{* * *}$ \\
6 & Indigirka & $0.29^{* * *}$ & $0.85^{* * *}$ & $-0.33^{* * *}$ & 19 & Pechora & $0.29^{* * *}$ & $0.87^{* * *}$ & $-0.32^{* * *}$ \\
7 & Indus & $0.29^{* * *}$ & $0.86^{* * *}$ & $-0.33^{* * *}$ & 20 & Senegal & $0.30^{* * *}$ & $0.83^{* * *}$ & $-0.33^{* * *}$ \\
8 & Kolyma & $0.27^{* * *}$ & $0.88^{* * *}$ & $-0.32^{* * *}$ & 21 & Volga & $0.26^{* * *}$ & $0.91^{* * *}$ & $-0.33^{* * *}$ \\
9 & Lena & $0.28^{* * * *}$ & $0.88^{* * *}$ & $-0.32^{* * *}$ & 22 & Yangtze & $0.29^{* * * *}$ & $0.85^{* * *}$ & $-0.34^{* * *}$ \\
10 & Mackenzie & $0.29^{* * * *}$ & $0.86^{* * *}$ & $-0.33^{* * *}$ & 23 & Yellow & $0.34^{* * * *}$ & $0.76^{* * *}$ & $-0.39^{* * *}$ \\
11 & Mississippi & $0.29^{* * * *}$ & $0.86^{* * *}$ & $-0.33^{* * *}$ & 24 & Yenisei & $0.26^{* * * *}$ & $0.90^{* * *}$ & $-0.32^{* * *}$ \\
12 & Niger & $0.29^{* * * *}$ & $0.86^{* * *}$ & $-0.33^{* * *}$ & 25 & Yukon & $0.29^{* * *}$ & $0.85^{* * *}$ & $-0.33^{* * *}$ \\
13 & Nile & $0.28^{* * * *}$ & $0.87^{* * *}$ & $-0.33^{* * *}$ & 26 & Amur & $0.30^{* * *}$ & $0.83^{* * *}$ & $-0.32^{* * *}$ \\
\hline
\end{tabular}

' $* * *$ ' represent the validated parameter are significant at the level of $p=0.01$ 
Table S3. The change points of runoff and the change rates of meteorological and vegetative factors after change points

\begin{tabular}{|c|c|c|c|c|c|c|c|c|c|}
\hline ID & Basin & $\begin{array}{c}\text { Changepoint } \\
\text { of } R\end{array}$ & $R$ & $E$ & $P e$ & PET & $n$ & $N D V I$ & $S I$ \\
\hline 1 & Amazon & 1998 & 8.5 & -1.0 & 3.4 & 1.1 & -9.4 & 3.4 & 0.3 \\
\hline 2 & Amur & 1998 & -16.4 & -0.3 & -5.8 & 3.0 & 4.5 & -1.3 & 24.9 \\
\hline 3 & Aral & 1994 & -14.8 & 12.8 & 5.2 & 3.8 & 12.4 & -0.8 & -6.1 \\
\hline 4 & Columbia & 1999 & -10.7 & 1.2 & -4.4 & 4.2 & 2.1 & -1.7 & 15.7 \\
\hline 5 & Congo & 1997 & 4.1 & -2.5 & -0.8 & 0.7 & -15.5 & 1.0 & 3.5 \\
\hline 6 & Danube & 1988 & -12.5 & 16.4 & 5.2 & 5.5 & 27.3 & 6.4 & 1.4 \\
\hline 7 & Indigirka & 1990 & -7.0 & 4.4 & -3.4 & 2.4 & 5.0 & 5.5 & 5.1 \\
\hline 8 & Indus & 1998 & -16.7 & -4.5 & -9.0 & 1.7 & 2.3 & 3.4 & 24.6 \\
\hline 9 & Kolyma & 1990 & -9.6 & 0.4 & -5.0 & 0.9 & 3.7 & 4.2 & 16.9 \\
\hline 10 & Lena & 1995 & 14.3 & 4.7 & 9.2 & -1.3 & 0.3 & 1.1 & -3.8 \\
\hline 11 & Mackenzie & 1989 & -13.3 & 6.2 & -3.5 & 2.3 & 10.5 & -2.7 & 13.1 \\
\hline 12 & Mississippi & 1998 & -20.1 & 5.0 & -2.0 & 0.0 & 15.1 & 1.3 & 8.7 \\
\hline 13 & Niger & 1990 & 27.9 & 7.7 & 13.7 & 0.6 & -2.6 & 6.5 & -4.1 \\
\hline 14 & Nile & 1995 & 14.7 & 3.2 & 5.7 & 1.9 & -2.9 & 3.1 & 12.5 \\
\hline 15 & $\begin{array}{c}\text { Northern } \\
\text { Dvina }\end{array}$ & 2000 & -7.1 & 6.7 & -1.1 & 2.2 & 9.4 & 1.3 & 8.5 \\
\hline 16 & $\mathrm{Ob}$ & 1998 & 7.5 & 4.7 & 5.9 & 1.8 & 0.9 & -0.8 & -7.0 \\
\hline 17 & Olenek & 1988 & 13.9 & 10.7 & 12.6 & -1.9 & 4.5 & 6.2 & -20.5 \\
\hline 18 & Parana & 1998 & -6.6 & 2.0 & 0.1 & 1.6 & 4.6 & -1.1 & 2.9 \\
\hline 19 & Pearl & 1991 & 16.3 & 2.9 & 10.1 & -0.7 & -0.5 & -1.6 & 19.0 \\
\hline 20 & Pechora & 1990 & 20.4 & -3.9 & 11.1 & 0.7 & -10.2 & 2.7 & -12.4 \\
\hline 21 & Senegal & 1993 & 28.3 & 15.3 & 16.9 & 0.9 & 1.7 & 7.6 & -9.3 \\
\hline 22 & Volga & 1994 & -8.9 & 4.1 & -1.2 & 2.3 & 6.8 & 3.8 & 1.6 \\
\hline 23 & Yangtze & 2000 & -4.5 & 5.9 & -0.6 & 3.0 & 5.2 & -0.3 & -3.2 \\
\hline 24 & Yellow & 1990 & -10.1 & 3.2 & -0.3 & 2.9 & 5.1 & 2.6 & 24.2 \\
\hline 25 & Yenisei & 1996 & 2.1 & 3.9 & 3.1 & 1.1 & 2.3 & 1.6 & 12.1 \\
\hline 26 & Yukon & 1994 & -8.0 & -28.4 & -15.6 & 2.2 & -18.9 & -3.4 & 8.9 \\
\hline
\end{tabular}


Table S4. Contributions to the long-term mean changes of $R$ and $E$ from $P_{e}, \mathrm{SAI}, \mathrm{M}$ and $E_{0}$ changes.

\begin{tabular}{|c|c|c|c|c|c|c|c|c|c|}
\hline \multirow[b]{2}{*}{ ID } & \multirow[b]{2}{*}{ Basins } & \multicolumn{4}{|c|}{ Contributions to $R$ changes } & \multicolumn{4}{|c|}{ Contributions to $E$ changes } \\
\hline & & $\mathrm{P}$ & E0 & $\mathrm{M}$ & SSI & $\mathrm{P}$ & E0 & $\mathrm{M}$ & SSI \\
\hline 1 & Amazon & 63.7 & -10.1 & 25.5 & -0.7 & 19.8 & 22.3 & 55.4 & -2.5 \\
\hline 2 & Amur & -59.9 & -11.2 & 4.2 & 24.6 & -51.7 & 13.5 & 13.6 & 21.2 \\
\hline 3 & Aral & -13.2 & -9.3 & -21.4 & 56.1 & 33.9 & 7.0 & -10.1 & 48.9 \\
\hline 4 & Columbia & -69.3 & -15.5 & 4.0 & 11.2 & -44.5 & 28.1 & 11.5 & 15.9 \\
\hline 5 & Congo & 26.2 & -8.1 & -30.8 & 34.9 & -7.8 & 10.1 & -37.7 & 44.4 \\
\hline 6 & Danube & 17.3 & -19.0 & 59.4 & -4.4 & 17.8 & 18.9 & 51.1 & 12.2 \\
\hline 7 & Indigirka & -54.3 & -6.5 & 30.2 & -9.0 & -21.4 & 11.2 & 58.0 & 9.4 \\
\hline 8 & Indus & -82.8 & -3.8 & -4.2 & 9.1 & -74.7 & 5.6 & 15.1 & -4.6 \\
\hline 9 & Kolyma & -67.0 & -3.7 & -13.3 & 16.0 & -45.6 & 6.1 & 31.2 & -17.0 \\
\hline 10 & Lena & 94.7 & 3.8 & 0.7 & 0.8 & 85.3 & -10.6 & -0.7 & 3.5 \\
\hline 11 & Mackenzie & -54.1 & -6.2 & 16.5 & 23.3 & -20.1 & 10.7 & 64.3 & -4.8 \\
\hline 12 & Mississippi & -36.8 & -0.2 & -20.4 & 42.7 & -17.4 & 0.2 & 51.5 & -30.9 \\
\hline 13 & Niger & 79.1 & -1.6 & 15.9 & 3.5 & 81.4 & 1.4 & 15.6 & 1.6 \\
\hline 14 & Nile & 61.8 & -8.1 & -13.4 & 16.7 & 68.1 & 6.8 & -11.2 & 13.9 \\
\hline 15 & Northern Dvina & -29.0 & -11.7 & -19.8 & 39.6 & -6.1 & 15.4 & 39.3 & -39.2 \\
\hline 16 & $\mathrm{Ob}$ & 83.5 & -9.5 & -1.9 & 5.2 & 70.1 & 17.1 & 7.1 & -5.7 \\
\hline 17 & Olenek & 82.5 & 2.9 & 6.2 & 8.4 & 54.2 & -7.5 & 34.0 & -4.3 \\
\hline 18 & Parana & -25.0 & -29.2 & 24.7 & 21.1 & 2.2 & 38.1 & 27.0 & 32.7 \\
\hline 19 & Pearl & 96.4 & 2.2 & 0.3 & 1.1 & 83.5 & -9.8 & 1.8 & 5.0 \\
\hline 20 & Pechora & 76.6 & -0.9 & 8.4 & 14.1 & 30.7 & 2.7 & 52.3 & -14.3 \\
\hline 21 & Senegal & 86.4 & -2.2 & 7.9 & 3.5 & 94.6 & 0.9 & 4.5 & 0.0 \\
\hline 22 & Volga & -41.3 & -13.5 & 39.6 & -5.6 & -12.0 & 20.2 & 49.6 & 18.1 \\
\hline 23 & Yangtze & -26.2 & -19.1 & -11.6 & 43.1 & -4.6 & 24.6 & -19.8 & 51.0 \\
\hline 24 & Yellow & -10.9 & -22.1 & -18.6 & 48.4 & -6.4 & 23.2 & -20.8 & 49.6 \\
\hline 25 & Yenisei & 60.7 & -10.0 & -8.7 & 20.6 & 42.2 & 14.7 & -11.4 & 31.7 \\
\hline 26 & Yukon & -63.8 & -1.3 & 19.6 & 15.3 & -25.7 & 2.6 & -20.8 & 50.9 \\
\hline
\end{tabular}

